\title{
ON COMMON FIXED POINTS, PERIODIC POINTS, AND RECURRENT POINTS OF CONTINUOUS FUNCTIONS
}

\author{
ALIASGHAR ALIKHANI-KOOPAEI \\ Received 15 May 2002 and in revised form 27 November 2002
}

\begin{abstract}
It is known that two commuting continuous functions on an interval need not have a common fixed point. However, it is not known if such two functions have a common periodic point. We had conjectured that two commuting continuous functions on an interval will typically have disjoint sets of periodic points. In this paper, we first prove that $S$ is a nowhere dense subset of $[0,1]$ if and only if $\left\{f \in C([0,1]): F_{m}(f) \cap \bar{S} \neq \varnothing\right\}$ is a nowhere dense subset of $C([0,1])$. We also give some results about the common fixed, periodic, and recurrent points of functions. We consider the class of functions $f$ with continuous $\omega_{f}$ studied by Bruckner and Ceder and show that the set of recurrent points of such functions are closed intervals.
\end{abstract}

2000 Mathematics Subject Classification: 54C50, 54C60, 54H25, 37E05, 26 A21.

1. Introduction. The subject of commuting continuous functions on an interval has been considered by a group of researchers in the 20th century. In the 1920s, J. F. Ritt in a sequence of papers investigated the algebraic properties of function composition as a binary operation on the set of rational complex functions. In particular, he showed that commuting polynomials always have a common fixed point. In 1954, Eldon Dyer asked whether two commuting continuous functions must have a common fixed point. The same question was asked by A. J. Shields in 1955 and L. Dubins in 1956. For sometimes, this conjecture was considered by a group of people and led them to some partial results. Boyce [4] and Huneke [6] answered this question in negative by the construction of a pair of commuting continuous functions which have no fixed point in common. In [2], we considered a generalization of the common fixedpoint problem and conjectured that in general two commuting continuous self-maps of the intervals do not have a common periodic point. To settle the conjecture, some partial results that gave more information about the structure of such pairs were obtained. However, in constructing such examples, we were convinced that the construction of such pair is extremely difficult, even though it seems that most pairs should not have any periodic points in common. Thus, in the Twenty-First Summer Symposium in Real Analysis, we posed 
Conjecture 1.1 and raised the possibility of using a Baire-category argument to settle the problem. It is also not difficult to show that commuting continuous self-maps of the intervals must have common recurrent points. However, this is not true in general for the case of metric spaces. In [1], we also constructed an example of a pair of commuting continuous self-maps of a compact metric space with no common periodic points, which also provided an answer to a question raised in [2].

CONJECTURE 1.1. Two commuting continuous functions on an interval will typically have disjoint sets of periodic points.

Steele [7] considered the above conjecture and investigated the likelihood of such pair $f$ and $g$ when $P(f)$ is first category and pointed out the difficulties that one might face using a Baire-category argument.

In this paper, we first provide a natural setting for the study of this problem and give some results including a generalization of Steele's main theorem. We also consider the class of functions with continuous $\omega_{f}(x)$ studied by Bruckner and Ceder [5] and show that the set of recurrent points of such functions is a closed interval. We begin with some preliminaries.

The class of continuous self-maps of a closed interval $I$ is denoted by $C(I, I)$. For $f \in C(I, I)$ and any integer $n \geq 1, f^{n}$ denotes the $n$th iterate of $f$. The orbit of $x$ under $f$ (i.e., the set $\left.\left\{f^{k}(x): k \geq 0\right\}\right)$ is denoted by $O(f, x)$. The set of cluster points of $O(f, x)$ is denoted by $\omega(f, x)$. We simply use $\omega(x)$ instead of $\omega(f, x)$ when there is no room for confusion. We also use $\omega_{f}(x)$ for the function $x \rightarrow \omega(f, x)$. A point $x$ is in $R(f)$ if $x \in \omega(f, x)$. Let $F_{n}(f)=$ $\left\{x \in I: f^{n}(x)=x\right\}$. We call $F(f)=F_{1}(f)$ the set of fixed points of $f, P(f)=$ $\cup_{n=1}^{\infty} F_{n}(f)$ the set of periodic points of $f$, and $R(f)$ the set of recurrent points of $f$, respectively. A subset $Y$ of $I$ is called invariant under $g$ if $g(Y) \subseteq Y$. A closed, invariant, nonempty subset of $I$ is called minimal if it contains no proper subset that is also closed, invariant, and nonempty. Every closed, invariant, and nonempty subset of $I$ contains a minimal set. If $Y$ is a minimal set, then $Y \subseteq R(f)$, and if it is not the orbit of a periodic point, then it is a perfect set. A minimal set is also nowhere dense.

Suppose $\mathscr{H}=\{(f, g): f, g \in C(I, I)\}$. Define $\rho: \mathscr{H} \rightarrow[0, \infty)$ by $\rho\left(\left(f_{1}, g_{1}\right)\right.$, $\left.\left(f_{2}, g_{2}\right)\right)=\left\|f_{1}-f_{2}\right\|+\left\|g_{1}-g_{2}\right\|$ where $\|f-g\|=\sup \{|f(x)-g(x)|: x \in I\}$. The open ball about $x$ with radius $\epsilon$ is denoted by $B_{\epsilon}(x)$, and the interior and closure of $A$ are denoted by $A^{o}$ and $\bar{A}$, respectively. The set of rational numbers is denoted by $\mathbb{Q}$ and the set of natural numbers is denoted by $\mathbb{N}$. For two compact subsets $A$ and $B$ of $I$, by $d(A, B)$ and $d_{H}(A, B)$, we mean the distance of the sets $A$ and $B$ with respect to the ordinary metric and Hausdorff metric, respectively. Even though the results given here are true on any closed interval $I$, we only prove them for $I=[0,1]$. In approximating a continuous function with a polynomial, we consider its Bernstein polynomials restricted to $I$. These polynomials have the interesting property that for $f \in C(I, I)$ all 
its Bernstein polynomials are also in $C(I, I)$. In the sequel, we use Theorem 1.2 given below from [3]. The proof of Theorem 1.3 is also trivial, and thus, it is omitted.

THEOREM 1.2. If $f \in C(I, I)$, then $\overline{P(f)}=\overline{R(f)}$.

THEOREM 1.3. Let $\mathscr{H}$ and $\rho$ be as defined, then $(\mathscr{H}, \rho)$ is a compact metric space.

\section{Main results}

LEMmA 2.1. Let $f \in C(I, I)$ and let $S$ be an $F_{\sigma}$ subset of $I$. Then $S$ is of first category if and only if $S$ does not contain an interval.

Proof. Suppose $S$ is of first category and $S=\cup_{m=1}^{\infty} S_{m}$ with each $S_{m}$ closed. Then, from the Baire-category theorem, it follows that $S$ does not contain an interval. On the other hand, if $S$ does not contain an interval, then for each $m \geq 1, S_{m}$ is a nowhere dense set, implying that $S$ is a first-category subset of $I$.

COROLLARY 2.2. Let $f \in C(I, I)$. Then $P(f)$ is of first category if and only if $P(f)$ does not contain an interval.

THEOREM 2.3. The set $A=\{(f, g): P(f)$ and $P(g)$ are both first category $\}$ is a residual subset of $\mathscr{H}$.

Proof. Let $A_{1}=\{(f, g) \in \mathscr{H}: P(f)$ is not first category $\}$ and let $A_{2}=$ $\{(f, g) \in \mathscr{H}: P(g)$ is not first category $\}$, then we have $A=\mathscr{H} \backslash\left(A_{1} \cup A_{2}\right)$. We show that $A$ is a residual subset of $\mathscr{H}$ by showing that $A_{1}$ and $A_{2}$ are firstcategory subsets of $\mathscr{H}$. We prove our claim for set $A_{1}$; the case for $A_{2}$ similarly follows. Suppose $(f, g) \in A_{1}$, then $P(f)$ is not of first category in $I$. Thus it contains an interval, hence we have

$$
\begin{aligned}
A_{1} & =\{(f, g) \in \mathscr{H}: P(f) \text { is not first category }\} \\
& =\{(f, g) \in \mathscr{H}: P(f) \text { contains an interval }\} \\
& =\bigcup_{m=1}^{\infty}\left\{(f, g) \in \mathscr{H}: F_{m}(f) \text { contains an interval }\right\} .
\end{aligned}
$$

Thus it suffices to show that, for each $m \geq 1$, the set $B_{m}=\{(f, g) \in \mathscr{H}$ : $F_{m}(f)$ contains an interval $\}$ is a first-category subset of $\mathscr{H}$. This is achieved if we show that $B_{m, n}=\left\{(f, g) \in \mathscr{H}: \mathbb{Q}_{n} \subseteq F_{m}(f)\right\}$ is a nowhere dense subset of $\mathscr{H}$, where $\left\{\mathbb{Q}_{n}\right\}$ is an enumeration of the rational intervals $(a, b)$ with $a, b \in \mathbb{Q} \cap[0,1]$. Suppose $\left(f_{1}, g_{1}\right) \in B_{m, n}$ and $\epsilon>0$, then we have $\mathbb{Q}_{n} \subseteq F_{m}\left(f_{1}\right)$. Let $P$ be a polynomial with $\left\|f_{1}-P\right\|<\epsilon$, then $\left(P, g_{1}\right) \in B_{\epsilon}\left(\left(f_{1}, g_{1}\right)\right)$. It is easy to see that $B_{m, n}$ is a closed subset of $\mathscr{H}$ and $F_{m}(P)$ has finitely many elements for 
a polynomial $P$, thus $F_{m}(P)$ does not contain $\mathbb{Q}_{n}$. This implies that $\overline{B_{m, n}}=B_{m, n}$ has no interior, hence $B_{m, n}$ is a nowhere dense subset of $\mathscr{H}$.

LEMMA 2.4. Let $S \subset[0,1]$ be a nowhere dense set and let $m$ be a positive integer. Then the set $A_{m}=\left\{f \in C([0,1]): F_{m}(f) \cap \bar{S} \neq \varnothing\right\}$ is a nowhere dense subset of $C([0,1])$.

Proof. First we show that $A_{m}$ is a closed subset of $C([0,1])$. For this, let $\left\{g_{n}\right\} \subseteq A_{m}$ be a sequence and $\left\{g_{n}\right\}$ converges uniformly to $g$; we show that $g \in A_{m}$. Since $F_{m}\left(g_{n}\right) \cap \bar{S} \neq \varnothing$, take $y_{n} \in F_{m}\left(g_{n}\right) \cap \bar{S}$. Without loss of generality, we may assume $\lim _{n \rightarrow \infty} y_{n}=y_{0}$, thus $y_{0} \in \bar{S}$. Let $\epsilon>0$ be arbitrary. Choose $k$ large enough so that $\left|g^{m}\left(y_{k}\right)-g^{m}\left(y_{0}\right)\right|<\epsilon,\left\|g_{k}^{m}-g^{m}\right\|<\epsilon$, and $\left|y_{k}-y_{0}\right|<\epsilon$. Then we have

$$
\begin{aligned}
\left|g^{m}\left(y_{0}\right)-y_{0}\right| & \leq\left|g^{m}\left(y_{0}\right)-g^{m}\left(y_{k}\right)+g^{m}\left(y_{k}\right)-g_{k}^{m}\left(y_{k}\right)+g_{k}^{m}\left(y_{k}\right)-y_{0}\right| \\
& \leq\left|g^{m}\left(y_{0}\right)-g^{m}\left(y_{k}\right)\right|+\left|g^{m}\left(y_{k}\right)-g_{k}^{m}\left(y_{k}\right)\right|+\left|g_{k}^{m}\left(y_{k}\right)-y_{0}\right| \\
& \leq\left|g^{m}\left(y_{0}\right)-g^{m}\left(y_{k}\right)\right|+|| g^{m}-g_{k}^{m}||+\left|y_{k}-y_{0}\right| \\
& \leq 3 \epsilon .
\end{aligned}
$$

Since $\epsilon$ was arbitrary, we have $g^{m}\left(y_{0}\right)=y_{0}$, implying that $y_{0} \in F_{m}(g) \cap \bar{S}$, hence $g \in A_{m}$. To show that $A_{m}$ is nowhere dense subset of $C([0,1])$, we show that for each $f \in A_{m}$ and $\epsilon>0$, there exists a function $h \in C([0,1])$ so that $\|h-f\|<\epsilon$ and $F_{m}(h) \cap \bar{S}=\varnothing$. To this end, let $P$ be a Bernstein polynomial of $f$ so that $\|f-P\|<\epsilon / 4 m$ and let $E_{1}=F_{1}(P) \cap \bar{S}=\left\{z_{1}, z_{2}, \ldots, z_{k}\right\}$. Due to the uniform continuity of $P$, for $\epsilon>0$, there exists $\delta_{k}>0$ so that for $t_{1}, t_{2} \in[0,1]$, with $\left|t_{1}-t_{2}\right|<4 \delta_{k}$, we have $\left|P\left(t_{1}\right)-P\left(t_{2}\right)\right| \leq \epsilon / 4 m k$. Let $\delta_{k}^{\prime}=(1 / 4) \min \left\{\mid z_{i}-\right.$ $z_{j} \mid$ for $\left.i \neq j, \delta_{k}, \epsilon / 4\right\}$. For each $z_{i} \in E_{1}$, we have one of the following cases.

CASE 1. For all $x \in\left(z_{i}-\delta_{k}^{\prime}, z_{i}\right) \cup\left(z_{i}, z_{i}+\delta_{k}^{\prime}\right), P(x)>x$.

CASE 2. For all $x \in\left(z_{i}-\delta_{k}^{\prime}, z_{i}\right) \cup\left(z_{i}, z_{i}+\delta_{k}^{\prime}\right), P(x)<x$.

CASE 3. For all $x \in\left(z_{i}-\delta_{k}^{\prime}, z_{i}\right), P(x)>x$, and for all $x \in\left(z_{i}, z_{i}+\delta_{k}^{\prime}\right)$, $P(x)<x$.

CASE 4. For all $x \in\left(z_{i}-\delta_{k}^{\prime}, z_{i}\right), P(x)<x$, and for all $x \in\left(z_{i}, z_{i}+\delta_{k}^{\prime}\right)$, $P(x)>x$.

We only prove Cases 1 and 3. Cases 2 and 4 are proved similarly.

In Case 1 , choose $x_{1}$ and $x_{2}$ so that $z_{1}-\delta_{k}^{\prime}<x_{1}<z_{1}<x_{2}<z_{1}+\delta_{k}^{\prime}$ and take $P_{1}(x)=P(x)$ for $x \leq x_{1}$ or $x \geq x_{2}$, being linear on the interval $\left[x_{1}, x_{2}\right.$ ].

In Case 3, choose $x_{1}, x_{2}$, and $x_{3}$ so that $z_{1}-\delta_{k}^{\prime}<x_{3}<z_{1}<x_{2}<x_{1}<z_{1}+\delta_{k}^{\prime}$, $P\left(x_{3}\right)-\epsilon / 8 m k<\left(x_{1}+x_{2}\right) / 2<P\left(x_{3}\right)+\epsilon / 8 m k$, and $\left[x_{2}, x_{1}\right] \cap \bar{S}=\varnothing$. In this case, take $P_{1}(x)=P(x)$ for $x \leq x_{3}$ or $x \geq x_{1}, P_{1}\left(\left(x_{1}+x_{2}\right) / 2\right)=\left(x_{1}+x_{2}\right) / 2$, being linear on the intervals $\left[x_{3},\left(x_{1}+x_{2}\right) / 2\right]$ and $\left[\left(x_{1}+x_{2}\right) / 2, x_{1}\right]$. Therefore we have $F_{1}\left(P_{1}\right) \cap \bar{S}=F_{1}(P) \backslash\left\{z_{1}\right\}$ and $\left\|P_{1}-P\right\| \leq \epsilon / 4 m k$. Repeating this process for each $z_{i} \in E_{1}, i \geq 2$, we can choose piecewise polynomials $P_{i}$ in $C([0,1])$ so that $\left\|P_{i}-P_{i-1}\right\| \leq \epsilon / 4 m k$ and $F_{1}\left(P_{i}\right) \cap \bar{S}=F_{1}(P) \backslash\left\{z_{1}, z_{2}, z_{3}, \ldots, z_{i}\right\}$. By taking 
$\mathbb{Q}_{1}=P_{k}$, we have $\left\|\mathbb{Q}_{1}-f\right\| \leq\left\|\mathbb{Q}_{1}-P_{k-1}\right\|+\left\|P_{k-1}-P_{k-2}\right\|+\cdots+\left\|P_{1}-P\right\|+\| P-$ $f \| \leq k(\epsilon / 4 m k)+\epsilon / 4 m=\epsilon / 2 m$ and $F_{1}\left(\mathbb{Q}_{1}\right) \cap \bar{S}=\varnothing$. Since $\mathbb{Q}_{1}$ is a continuous piecewise polynomial, $F_{2}\left(\mathbb{Q}_{1}\right)$ has finitely many elements. Thus, by replacing the role of $P$ with $\mathbb{Q}_{1}$ and the role of $F_{1}(P)$ with $F_{2}\left(\mathbb{Q}_{1}\right)$, similarly, we may choose a piecewise polynomial $\mathbb{Q}_{2}$ in $C([0,1])$ so that $\left\|\mathbb{Q}_{2}-\mathbb{Q}_{1}\right\| \leq 3 \epsilon / 4 m$ and $F_{2}\left(\mathbb{Q}_{2}\right) \cap \bar{S}=\varnothing$. Continuing this process, we may choose piecewise polynomials $\left\{\mathbb{Q}_{i}\right\}_{i=1}^{m} \subset C([0,1])$ so that $\left\|f-\mathbb{Q}_{1}\right\| \leq 3 \epsilon / 4 m,\left\|\mathbb{Q}_{i}-\mathbb{Q}_{i-1}\right\| \leq 3 \epsilon / 4 m$ for $2 \leq i \leq$ $m$, and $F_{i}\left(\mathbb{Q}_{i}\right) \cap \bar{S}=\varnothing$ for $1 \leq i \leq m$. Thus we have $\left\|\mathbb{Q}_{m}-f\right\| \leq\left\|\mathbb{Q}_{m}-\mathbb{Q}_{m-1}\right\|+$ $\left\|\mathbb{Q}_{m-1}-\mathbb{Q}_{m-2}\right\|+\cdots+\left\|\mathbb{Q}_{1}-f\right\| \leq 3 \epsilon / 4 m+3 \epsilon / 4 m+\cdots+3 \epsilon / 4 m+3 \epsilon / 4 m \leq$ $m(3 \epsilon / 4 m)=3 \epsilon / 4<\epsilon$, and $F_{m}\left(\mathbb{Q}_{m}\right) \cap \bar{S}=\varnothing$. This implies that $\mathbb{Q}_{m} \notin A_{m}$ and hence $A_{m}$ is a nowhere dense subset of $C([0,1])$.

LEMMA 2.5. Let $S \subseteq[0,1]$ and let $m$ be a positive integer. If the set $A_{m}=$ $\left\{f \in C([0,1]): F_{m}(f) \cap \bar{S} \neq \varnothing\right\}$ is a nowhere dense subset of $C([0,1])$, then $S$ is a nowhere dense subset of $[0,1]$.

Proof. On the contrary, suppose $S$ is somewhere dense. Then $\bar{S}$ contains a closed interval $[a, b]$ of positive length. Define the function $f$ as $f(x)=x$, for $x \leq a$ or $x \geq b, f(a+(b-a) / 8)=1, f(a+(b-a) / 4)=a+(b-a) / 4$, $f(x)=x$, for $a+(b-a) / 4 \leq x \leq a+3(b-a) / 8, f(a+(b-a) / 2)=0$ and linear elsewhere. Take $\epsilon<(1 / 2) \min \{1-(a+(b-a) / 4), a+(b-a) / 2\}$. It is easy to see that for each $g \in C([0,1])$ with $\|g-f\|<\epsilon$, there exist $x_{1}, x_{2} \in(a, b)$ so that $g\left(x_{1}\right)>x_{1}, g\left(x_{2}\right)<x_{2}$. This implies the existence of $x_{3} \in(a, b) \cap F_{1}(g) \subseteq$ $F_{m}(g) \cap \bar{S}$, hence $g \in A_{m}$, contrary to the assumption that $A_{m}$ is a nowhere dense subset of $C([0,1])$.

By combining Lemmas 2.4 and 2.5, we have the following theorem.

THEOREM 2.6. Let $S \subseteq[0,1]$ and let $m$ be a positive integer. Then the set $A_{m}=\left\{f \in C([0,1]): F_{m}(f) \cap \bar{S} \neq \varnothing\right\}$ is a nowhere dense subset of $C([0,1])$ if and only if $S$ is a nowhere dense subset of $[0,1]$.

THEOREM 2.7. Let $S \subset[0,1]$ be a first-category set. Then there exists a residual subset $M$ of $C([0,1])$ so that $P(g) \cap S=\varnothing$ for each $g \in M$.

Proof. Let $S=\cup_{n=1}^{\infty} S_{n}$, where $S_{n}$ is nowhere dense for each $n$. For each positive integer $m$ from Theorem 2.6, we have that the set $A_{m, n}=\{g \in C([0,1])$ : $\left.F_{m}(g) \cap \overline{S_{n}} \neq \varnothing\right\}$ is a nowhere dense subset of $C([0,1])$. Thus $B=\cup_{m=1}^{\infty} \cup_{n=1}^{\infty}$ $A_{m, n}$ is a first-category subset of $C([0,1])$. Let $M=C([0,1]) \backslash B$, then $M$ is residual and if $g \in M$, then $g \notin A_{m, n}$ for each $m \geq 1$ and $n \geq 1$. Thus we have $F_{m}(g) \cap \overline{S_{n}}=\varnothing$ for $m \geq 1$ and $n \geq 1$. This implies that $S \cap P(g)=\varnothing$ for each $g \in M$.

THEOREM 2.8. The function $h: \mathscr{H} \rightarrow[0, \infty)$ defined by $h((f, g))=d(F(f), F(g))$ is a lower semicontinuous function.

Proof. The proof is straightforward and is omitted. 
COROLlary 2.9. Let $\mathscr{A}=\{(f, g) \in \mathscr{H}: f$ and $g$ have a common fixed point $\}$ and let $\mathscr{B}=\{(f, g) \in \mathscr{H}: f$ and $g$ have a common periodic point $\}$. Then $\mathscr{A}$ and $\mathscr{B}$ are closed and $F_{\sigma}$ subsets of $\mathscr{H}$, respectively.

Now we turn our attention to commuting continuous functions on the interval and prove the following lemma that is used in Theorem 2.11.

LEMmA 2.10. Let $f \in C(I, I)$ where $I=[0,1]$, and let $a, b \in I, a \neq b$. If $\epsilon>0$ is a real number, then there exists a function $h \in C(I, I)$ such that $h(a)=f(a)$, $h(b) \neq f(b)$, and $\|h-f\|<\epsilon$.

Proof. We can assume $\epsilon<1$. Then, for $x \in I=[0,1]$, let

$$
h(x)=f(x)+(c-f(x))|x-a| c \epsilon,
$$

with $c=1 / 2$ if $f(b) \neq 1 / 2$ and $c=1 / 3$ if $f(b)=1 / 2$. Then $h$ is continuous, $h(a)=f(a), h(b) \neq f(b)$, and $|h(x)-f(x)|=|(c-f(x))| x-a|c \epsilon<| x-$ $a \mid(\epsilon / 2) \leq \epsilon / 2$ for any $x \in I$. Thus, $\|h-f\|<\epsilon$. Moreover, $h(x) \in[0,1]$ for $x \in I$. If $f(x) \geq c, 0 \leq h(x) \leq f(x)$, and $f(x) \leq h(x) \leq 1$ if $f(x)<c$. Thus $h \in C(I, I)$.

THEOREM 2.11. Let $\mathscr{E}=\{(f, g) \in \mathscr{H}: f \circ g=g \circ f\}$, then $\mathscr{E}$ is a nonempty, closed, and nowhere dense subset of $\mathcal{H}$.

Proof. It is easy to see that $(f, f) \in \mathscr{L}$ when $f$ is the identity function, so $\mathscr{L}$ is nonempty. Suppose $\left(f_{n}, g_{n}\right) \in \mathscr{L}$ and $\left(f_{n}, g_{n}\right) \rightarrow(f, g)$. Then we have $f_{n} \rightarrow f, g_{n} \rightarrow g$, and $f_{n} \circ g_{n}=g_{n} \circ f_{n}$ for each $n$. We show that $(f, g) \in$ $\mathscr{L}$. Let $x \in I$ and $\epsilon>0$. Since $f$ and $g$ are uniformly continuous, we may choose $\delta$, so that $0<\delta<\epsilon$ and for every $x_{1}, x_{2}$ in $I$ with $\left|x_{1}-x_{2}\right|<\delta$, we have $\left|f\left(x_{1}\right)-f\left(x_{2}\right)\right|<\epsilon,\left|g\left(x_{1}\right)-g\left(x_{2}\right)\right|<\epsilon$. Since $f_{n} \rightarrow f$ and $g_{n} \rightarrow g$, there exists $N \in \mathbb{N}$ such that $n>N$ implies $\left\|f_{n}-f\right\|<\delta,\left\|g_{n}-g\right\|<\delta$. Thus, if $n>N,|f(g(x))-g(f(x))| \leq\left|f(g(x))-f\left(g_{n}(x)\right)\right|+\mid f\left(g_{n}(x)\right)-$ $f_{n}\left(g_{n}(x)\right)|+| g_{n}\left(f_{n}(x)\right)-g\left(f_{n}(x)\right)|+| g\left(f_{n}(x)\right)-g(f(x)) \mid \leq 2 \delta+2 \epsilon \leq 4 \epsilon$. Since $\epsilon>0$ and $x$ were arbitrary, we have $f \circ g=g \circ f$, so $(f, g) \in \mathscr{L}$ and $\mathscr{L}$ is closed. Now we show that $\mathscr{Z}$ is nowhere dense. Let $\epsilon>0,(f, g) \in \mathscr{L}$. Suppose $f$ or $g$ is not the identity function, hence there exists $x_{0}$ so that $f\left(x_{0}\right) \neq x_{0}$ or $g\left(x_{0}\right) \neq x_{0}$. Suppose $g\left(x_{0}\right) \neq x_{0}$, the case where $f\left(x_{0}\right) \neq x_{0}$ is similar. Let $h_{2}(x)=g(x)$ for each $x \in I$, and let $h_{1} \in C(I, I)$ be a function so that $h_{1}\left(x_{0}\right)=f\left(x_{0}\right)$, but $h_{1}\left(g\left(x_{0}\right)\right) \neq f\left(g\left(x_{0}\right)\right)$ and $\left\|f-h_{1}\right\|<\epsilon$. From Lemma 2.10, with $a=x_{0}$ and $b=g\left(x_{0}\right)$, it follows that such $h_{1} \in C(I, I)$ exists. Then we have $\left(h_{1}, h_{2}\right) \in B_{\epsilon}((f, g))$, and $h_{2}\left(h_{1}\left(x_{0}\right)\right)=h_{2}\left(f\left(x_{0}\right)\right)=g\left(f\left(x_{0}\right)\right)=$ $f\left(g\left(x_{0}\right)\right) \neq h_{1}\left(g\left(x_{0}\right)\right)=h_{1}\left(h_{2}\left(x_{0}\right)\right)$. Thus $\mathscr{L}$ is the union of a nowhere dense set and a singleton $\{(x, x)\}$, thus it is a nowhere dense subset of $\mathscr{H}$.

THEOREM 2.12. Let $f$ and $g$ be two commuting continuous self-maps of the unit interval. Then we have one of the following holds: 
(i) $f$ and $g$ have a common periodic point;

(ii) for any two positive integers $m$ and $n$, let $A_{m, n}=\left\{x: f^{n}(x)=g^{m}(x)\right\}$. Then for all $x \in A_{m, n}, \omega(f, x)$ is a perfect nowhere dense set.

Proof. The set $A_{m, n}$ is not empty as is shown in [2], however we give the proof for completeness. If $A_{m, n}$ is empty, the continuity of $f$ and $g$ permits us to assume without loss of generality that

$$
f^{n}(x)<g^{m}(x) \quad \forall x \in I .
$$

Since $g^{m}(1) \leq 1$, the set $S=\left\{x \in I: g^{m}(x) \leq x\right\}$ is not empty. Thus, since $S$ is closed, $S$ has a minimum element $c$. Clearly, $c=g^{m}(c)$. Hence $f^{n}(c)=$ $f^{n}\left(g^{m}(c)\right)=g^{m}\left(f^{n}(c)\right)$ so that $f^{n}(c) \in S$. Consequently, $f^{n}(c) \geq c=g^{m}(c)$. Since $f^{n}(c) \geq g^{m}(c)$ contradicts (2.4), the assertion that $A_{m, n}$ is empty is false. Now suppose that (i) does not hold, that is, $f$ and $g$ do not have a common periodic point, and let $x \in A_{m, n}$. Then $f^{n}(x)=g^{m}(x)$, and hence $f^{n}(f(x))=$ $f\left(g^{m}(x)\right)=g^{m}(f(x))$ and $f^{n}(g(x))=g\left(f^{n}(x)\right)=g\left(g^{m}(x)\right)=g^{m}(g(x))$. Thus if $x \in A_{m, n}, f(x), g(x) \in A_{m, n}$, and $f^{p n}(x)=g^{p m}(x)$ for each positive integer $p$. From this, it follows that $O(g, x)$ is contained in $A_{m, n}$ whenever $x \in A_{m, n}$. From closeness of $A_{m, n}$, it follows that $\omega(g, x) \subseteq A_{m, n}$. If $\omega(g, x)$ has an isolated point, then the isolated point is a periodic point of $g$ which is contained in $A_{m, n}$, thus also a periodic point of $f$, which is a contradiction. So for each $x \in A_{m, n}, \omega(g, x)$ is a perfect set. Suppose for some $x \in A_{m, n}, \omega(g, x)$ is somewhere dense, then it contains an interval $I_{0}$. Let $I_{1}$ be the largest interval such that $I_{0} \subseteq I_{1} \subseteq A_{m, n}$ and $x_{0} \in I_{1}^{o}$, then there are positive integers $k_{1}, k_{2}$ with $k_{1}<k_{2}$ so that $g^{k_{1}}(x)$ and $g^{k_{2}}(x)$ are in $I_{1}$. This implies that $g^{k_{2}-k_{1}}\left(I_{1}\right) \subseteq I_{1}$, and thus $g$ should have a periodic point in $I_{1}$ that is also a periodic point of $f$, which is again a contradiction. It is also easy to see that the set $\omega(g, x)$ is an invariant set under $g$. Suppose $A_{1}$ is a minimal set contained in $\omega(g, x)$. Since $f$ and $g$ have no common periodic points, the set $A_{1}$ cannot be the orbit of a periodic point. Hence $A_{1}$ is also a perfect set contained in $A_{m, n} \cap \omega(g, x)$. Thus, for each $x \in A_{m, n}, \omega(g, x)=\omega(f, x)$ is a perfect nowhere dense set containing a perfect minimal set.

REMARK 2.13. Bruckner and Ceder [5] proved that $\omega_{f}$ is Baire 1 if and only if any infinite $\omega$-limit set for $f$ is perfect. Motivated by this result, one may extend the above Baire- 1 classification of $\omega_{f}$ to the closed subsets of $I$. If so, the above theorem tells us either the two commuting continuous functions $f$ and $g$ have a common periodic point or both $\omega_{f}$ and $\omega_{g}$ are Baire 1 on the closed set $A_{m, n}$ with $\omega(f, x)$ and $\omega(g, x)$ being nowhere dense for each $x \in A_{m, n}$.

REMARK 2.14. An argument similar to the proof of the above theorem could give the same result if the role of $A_{m, n}$ is replaced with any nonempty $f$-invariant closed subset of $P(g)$ or $g$-invariant closed subset of $P(f)$. In 
particular, Theorem 2.12 is also true if in its statement we replace $A_{m, n}$ with $F(f)$ or $F(g)$.

The family of all $\omega$-limit sets of a continuous function with the Hausdorff metric forms a metric space. Bruckner and Ceder [5] introduced a kind of chaos in terms of the map $\omega_{f}: x \rightarrow \omega(f, x)$. In the same paper, they proved Lemma 2.15 and used it to prove Theorem 2.16 which characterizes the continuity of $\omega_{f}$.

LEMMA 2.15. If $\omega_{f}$ is continuous, then $F_{1}(f)$ is connected.

THEOREM 2.16. The following conditions are equivalent:

(1) $\omega_{f}$ is continuous;

(2) $\left\{f^{n}\right\}_{n=1}^{\infty}$ is equicontinuous;

(3) $\omega_{f^{2}}$ is continuous;

(4) $F_{1}\left(f^{2}\right)=\cap_{n=1}^{\infty} f^{n}(I)$;

(5) $F_{1}\left(f^{2}\right)$ is connected and, for all $x,\left\{f^{2 n}(x)\right\}_{n=1}^{\infty}$ converges to a point of $F_{1}\left(f^{2}\right)$;

(6) $F_{1}\left(f^{2}\right)$ is connected;

(7) $\omega_{f}$ is lower semicontinuous;

(8) $\omega_{f}$ is upper semicontinuous.

Using the above theorem, we show that the recurrent set of a continuous function defined on a closed interval with continuous $\omega_{f}$ is a closed interval.

LEMмA 2.17. For any natural number $n, \omega_{f n}$ is continuous when $\omega_{f}$ is continuous.

Proof. From the lower semicontinuity of $\omega_{f}$, it follows that $\left\{f^{k}\right\}_{k=1}^{\infty}$ is equicontinuous, implying that $\left\{f^{n k}\right\}_{k=1}^{\infty}$ is equicontinuous for each $n \in \mathbb{N}$. Hence $\omega_{f}$ is also lower semicontinuous. Thus the lemma follows from Theorem 2.16.

THEOREM 2.18. Suppose $f$ is a continuous self-map of the unit interval with $\omega_{f}: x \rightarrow \omega(f, x)$ continuous. Then $R(f)$ is a closed interval.

Proof. For each $m \geq 1, \omega_{f m}$ is continuous by Lemma 2.17, thus $F_{1}\left(f^{m}\right)=$ $F_{m}(f)$ is connected by Lemma 2.15. We also have $F_{1}(f) \subseteq F_{n}(f)$ for all $n \geq 1$, implying that $\varnothing \neq F_{1}(f) \subseteq \bigcap_{n=1}^{\infty} F_{n}(f)$, thus $P(f)=\bigcup_{n=1}^{\infty} F_{n}(f)$ is connected. Since $P(f) \subseteq R(f) \subseteq \overline{P(f)}$, we have $R(f)$ connected. Now let $\left\{x_{n}\right\}_{n=1}^{\infty}$ be a sequence in $R(f)$ with $\lim _{n \rightarrow \infty} x_{n}=x_{0}$. We show that $x_{0} \in R(f)$. Let $\epsilon$ be an arbitrary positive number. From the continuity of $\omega_{f}$ and the fact that $\lim _{n \rightarrow \infty} x_{n}=$ $x_{0}$, there exists $N_{1} \in \mathbb{N}$ so that $\left|x_{n}-x_{0}\right|<\epsilon$ and $d_{H}\left(\omega\left(f, x_{n}\right), \omega\left(f, x_{0}\right)\right)<\epsilon$ for all $n \geq N_{1}$. Since $\left\{x_{n}\right\} \subseteq R(f)$, we have $x_{n} \in \omega\left(f, x_{n}\right)$ for each $n$. Thus, for each $n \geq N_{1}$, there exists $z_{n} \in \omega\left(f, x_{0}\right)$ so that $\left|x_{n}-z_{n}\right|<\epsilon$, implying that $\left|z_{n}-x_{0}\right|<2 \epsilon$. Hence $d\left(x_{0}, \omega\left(f, x_{0}\right)\right) \leq 2 \epsilon$, for every $\epsilon>0$, implying that $x_{0} \in \omega\left(f, x_{0}\right)$, hence $x_{0} \in R(f)$. Thus we have shown that $R(f)$ is a closed and connected subset of the real line, hence it is a closed interval. 
REMARK 2.19. Due to the fact that $R(f)=\cap_{m=1}^{\infty} \cup_{n=m}^{\infty}\left\{x \in I: \mid f^{n}(x)-\right.$ $x \mid \leq 1 / m\}$, we see that the recurrent set of continuous self-maps of a closed interval is an $F_{\sigma \delta}$ set. A question of considerable interest is the classification of such maps with $F_{\sigma}$ recurrent set. This class of functions contains nomadic functions. A continuous self-map of an interval is called nomadic if it has a dense orbit at some point. It is also known that if $R(f)$ is closed, then $f$ has topological entropy zero. Thus Theorem 2.18 provides another subclass of the class of continuous self-maps of the interval with an $F_{\sigma}$ recurrent set as well as zero topological entropy.

ACKNOWLEDGment. I wish to thank the anonymous referee for his useful and important suggestions as well as for proposing the present version of Lemma 2.10 which is more general than our original version.

\section{REFERENCES}

[1] A. Alikhani-Koopaei, A counter example on common periodic points of functions, Int. J. Math. Math. Sci. 21 (1998), no. 4, 823-827.

[2] On common fixed and periodic points of commuting functions, Int. J. Math. Math. Sci. 21 (1998), no. 2, 269-276.

[3] L. S. Block and W. A. Coppel, Dynamics in One Dimension, Lecture Notes in Mathematics, vol. 1513, Springer-Verlag, Berlin, 1992.

[4] W. M. Boyce, Commuting functions with no common fixed point, Trans. Amer. Math. Soc. 137 (1969), 77-92.

[5] A. M. Bruckner and J. Ceder, Chaos in terms of the map $x \rightarrow \omega(x, f)$, Pacific J. Math. 156 (1992), no. 1, 63-96.

[6] J. P. Huneke, On common fixed points of commuting continuous functions on an interval, Trans. Amer. Math. Soc. 139 (1969), 371-381.

[7] T. H. Steele, A note on periodic points and commuting functions, Real Anal. Exchange 24 (1998/99), no. 2, 781-789.

Aliasghar Alikhani-Koopaei: Berks-Lehigh Valley College, Pennsylvania State University, Reading, PA 19610-6009, USA

E-mail address: axa12@psu.edu 


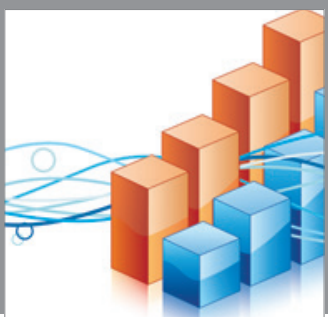

Advances in

Operations Research

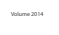

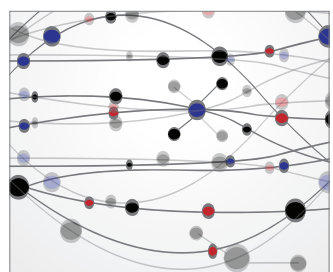

\section{The Scientific} World Journal
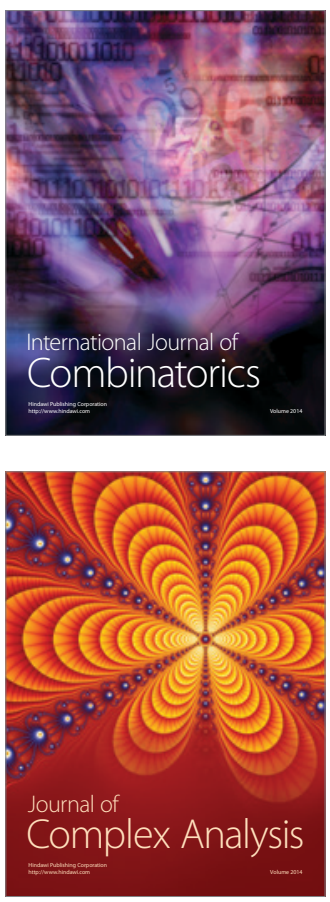

International Journal of

Mathematics and

Mathematical

Sciences
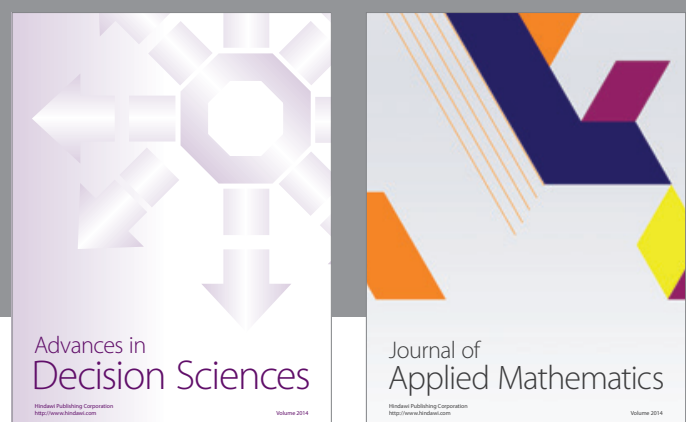

Journal of

Applied Mathematics
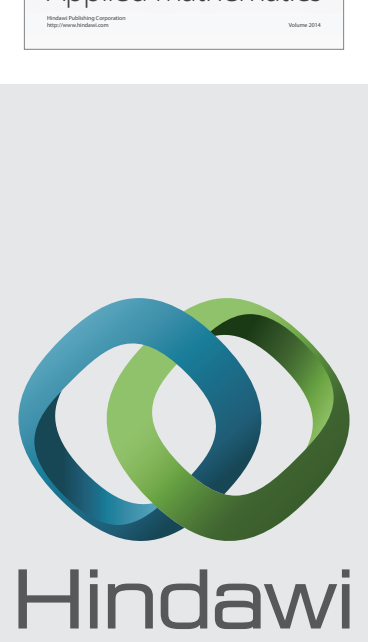

Submit your manuscripts at http://www.hindawi.com
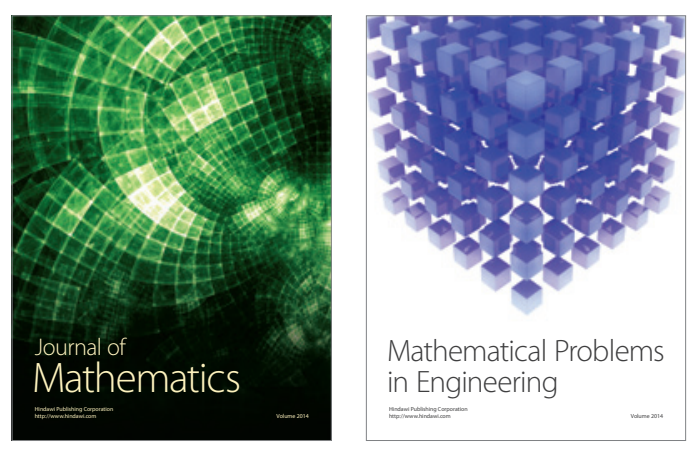

Mathematical Problems in Engineering
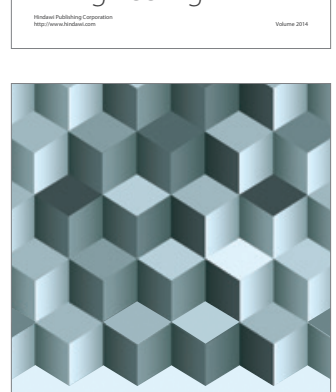

Journal of

Function Spaces
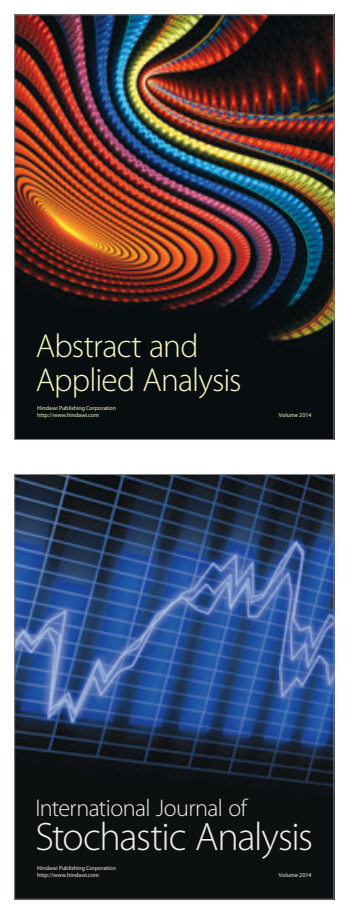

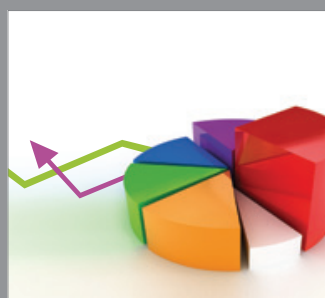

ournal of

Probability and Statistics

Promensencen
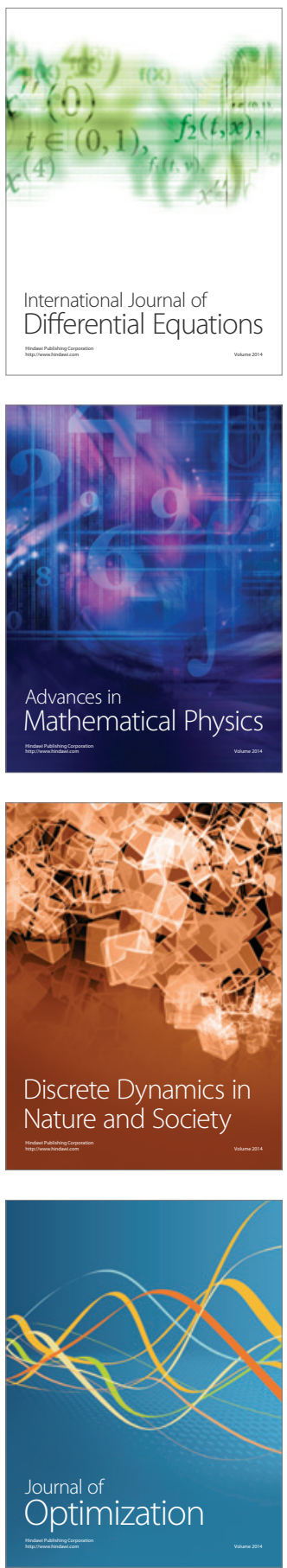